\title{
以 $\mathrm{ZnO}$ 为模板和催化剂将PVC转化为分级多孔 碳材料
}

\author{
孟甜甜 ${ }^{1}$, 章骅 $1^{1,2}$, 吕凡 ${ }^{1,2}$, 邵立明 ${ }^{1,2}$, 何品晶 ${ }^{1,2}$ \\ 1. 同济大学环境科学与工程学院, 上海 200092 \\ 2. 上海污染控制与生态安全研究院, 上海 200092 \\ *通讯作者, E-mail: zhanghua_tj@tongji.edu.cn \\ 收稿日期: 2021-06-23; 接受日期: 2021-09-15; 网络版发表日期: 2021-11-29 \\ 国家重点研发计划(编号: 2018YFD1100600)资助项目
}

摘要聚氯乙烯 $(\mathrm{PVC})$ 在 $250 \sim 310^{\circ} \mathrm{C}$ 时发生脱氯反应, 碳原子由 $\mathrm{sp}^{3}$ 杂化转变为 $\mathrm{sp}^{2}$ 杂化, 随着温度的升高, 这些 $\mathrm{sp}^{2}$ 杂化的碳原子进一步组装形成芳香烃类型的碳, 热解碳产物往往具有一定石墨化程度, 具有较高的导电性, 是 制作电容器电极的优良前驱物. 本研究在没有活化剂的情况下, 以纳米 $\mathrm{ZnO}$ 作为模板和催化剂, 热解PVC成功制 备了高产率的分级多孔碳材料(HPC), 比电容 $(1 \mathrm{~A} / \mathrm{g})$ 达到 $226 \mathrm{~F} / \mathrm{g}$. 通过比较 $\mathrm{ZnO}$ 和 PVC的混合方式, 以及高温碳化 前是否去除 $\mathrm{ZnCl}_{2}$, 发现 $\mathrm{ZnO}$ 会降低碳材料的石墨化程度, 同时在材料表面引入含氧官能团, 而 $\mathrm{ZnCl}_{2}$ 的作用与之相 反. 后续可通过控制催化剂的种类与尺寸, 改善 $\mathrm{HPC}$ 的孔结构和石墨化性质等增强其电容性能, 该研究为 PVC基 $\mathrm{HPC}$ 的设计提供了参考.

关键词聚氯乙烯, 纳米氧化锌, 模板碳化, 催化, 电容

\section{1 引言}

世界当前处于塑料时代，塑料的耐用、轻便和耐 腐蚀性使它们得到广泛应用. 2019年，全球塑料的生 产量达3.68亿吨 ${ }^{[1]}$ ，但大量消耗、快速废弃的塑料给 各国带来了严重的环境问题. 塑料废物累积量在 2015 年已达到63亿吨，其中约 $9 \%$ 被回收，12\%被焚烧，79\% 进入垃圾填埋场或自然环境中 ${ }^{[2]}$. 塑料内碳元素含量 很高, 是制备碳材料的理想前驱物. 近十几年来, 塑料 等聚合物碳化领域的研究蓬勃发展, 具有多级(含有微 孔、介孔、大孔中的两种或三种不同孔径的孔隙)孔
道体系的分级多孔碳材料(HPC)在阻燃、催化、吸附 分离、能源存储等领域展现出广阔的应用前景.

含卤素(如氟、氯、溴)塑料可能会长期释放有毒 物质, 对人类和/或环境更危险. 作为使用最广泛的含 卤素塑料与世界生产量第三的聚合物, $\mathrm{PVC}$ 的回收利 用被广泛关注. $\mathrm{PVC}$ 在 $250 \sim 310^{\circ} \mathrm{C}$ 时发生脱氯反应, 氯 原子与邻近碳原子上的氢结合, 生成氯化氢, 碳原子由 $\mathrm{sp}^{3}$ 杂化转变为 $\mathrm{sp}^{2}$ 杂化, 形成碳碳双键 ${ }^{[3]}$. 随着温度升 高, 这些 $\mathrm{sp}^{2}$ 杂化的碳原子进一步组装形成芳香烃类型 的碳, 热解碳产物往往具有一定石墨化程度 ${ }^{[4]}$, 具有较 高的导电性, 是制作电容器电极的优良前驱物.

引用格式: Meng TT, Zhang H, Lü F, Shao LM, He PJ. Convert PVC to hierarchical porous carbon material by using ZnO as a template and catalyst. Sci Sin Chim, 2022, 52: 482-493, doi: 10.1360/SSC-2021-0148 
然而, PVC直接热解时得到的固体残渣产量低, 是 一种黑色、易碎和多孔的材料 ${ }^{[3]}$, 比表面积较低, 难以 直接作为双电层电容器电极材料利用. 如何提高PVC 碳化的产率和控制其碳化产物的微观结构是一个挑 战. 近年来, 研究者通过模板碳化法将PVC转化为可用 作电容器电极的碳材料 ${ }^{[5]}$, 电容量在 $50 \mathrm{~F} \mathrm{~g}^{-1}$ 左右 ${ }^{[6]}$, 后 续仍需要采用氮掺杂 ${ }^{[4]}$ 、负载金属氧化物 ${ }^{[6]}$ 等方法提 高其电容量. 此外, 碱性物质如 $\mathrm{KOH}$ 等在球磨或热解 的过程中能有效地实现 PVC的脱氯 ${ }^{[7,8]}$, 但是碱性过强 的添加物会促进PVC分解, 降低碳的产率. 为此, 需要 一种合适的方法来处理PVC, 实现氯的回收、制备高 产率且具有定制形貌和结构的碳材料.

在PVC催化热解制油过程中, 许多催化剂如Al-Zn 复合材料 ${ }^{[9]} 、 \mathrm{ZnO}^{[10]} 、 \mathrm{Fe}_{2} \mathrm{O}_{3}{ }^{[11]}$ 等具有降低热解油产 量, 促进焦炭形成的效果. 因此, 本研究采用 $\mathrm{ZnO}$ 作为 催化剂和碳化模板, 通过一步和两步热解法探讨脱氯 与碳化反应的关系, 采用两种混合方式(干混法和溶剂 分散法)研究 $\mathrm{ZnO}$ 和PVC的接触对脱氯及碳化的影响.

\section{2 实验部分}

\section{1 碳材料制备}

将1 g PVC (K-value 68-85, 购自阿拉丁, 中国)加 入到 $10 \mathrm{~mL}$ 四氢呋喃(THF)溶剂中, 搅拌 $12 \mathrm{~h}$ 后形成透 明溶液. 然后, 加入 $2 \mathrm{~g}$ 平均直径约为 $7 \mathrm{~nm}$ 的 $\mathrm{ZnO}$ 粉末 ( $\mathrm{ZnOnm}$, 购自华赛纳米科技), 再摚拌 $24 \mathrm{~h}$, 得到 $\mathrm{ZnOnm}$ 在PVC和THF混合物中的均匀分散体. 然后将 $\mathrm{ZnOnm}-\mathrm{PVC}-\mathrm{THF}$ 分散体在 $60^{\circ} \mathrm{C}$ 烘箱中加热, 蒸发 $\mathrm{THF}$ 溶剂, 得到 $\mathrm{PVC}$ 包覆在 $\mathrm{ZnOnm}$ 上的复合材料, 将 其研磨成粉末, 即为溶剂分散反应物 S-PVC-ZnOnm, 将该方法称为溶剂分散法. 直接将 $1 \mathrm{~g} P \mathrm{PVC}$ 和 $2 \mathrm{~g}$ $\mathrm{ZnOnm}$ 在玛瑙研钵中研磨 $5 \mathrm{~min}$, 获得的混合物即为干 混反应物D-PVC-ZnOnm, 将该方法称为干混法.

分别采取一步法和两步法在管式炉内制备碳材 料. 一步法中, 将纯PVC、D-PVC-ZnOnm或S-PVC$\mathrm{ZnOnm}$ 以 $5^{\circ} \mathrm{C} / \mathrm{min}$ 的升温速率直接加热至 $800^{\circ} \mathrm{C}$, 保温 $2 \mathrm{~h}, \mathrm{~N}_{2}$ 流量为 $100 \mathrm{~mL} / \mathrm{min}$; 管式炉冷却至室温后, 将 反应产物在 $1 \mathrm{~mol} / \mathrm{L}$ 的盐酸溶液中浸出 $12 \mathrm{~h}$, 刻蚀其中 的ZnO, 浸出氯盐, 然后过滤、烘干. 纯PVC、D-PVCZnOnm 和S-PVC-ZnOnm采用一步法制备的碳材料分 别命名为 $O-P 、 O-D 、 O-S$.
在两步法中, 首先将D-PVC-ZnOnm或S-PVC$\mathrm{ZnOnm}$ 加热至 $310^{\circ} \mathrm{C}$, 保温 $0.5 \mathrm{~h}$, 升温速率为 $5^{\circ} \mathrm{C} / \mathrm{min}$, 氮气流量为 $100 \mathrm{~mL} / \mathrm{min}$; 固体残渣冷却后用水浸出 $12 \mathrm{~h}$, 然后过滤烘干; 将烘干的产物以 $5^{\circ} \mathrm{C} / \mathrm{min}$ 的升温 速率直接加热至 $800{ }^{\circ} \mathrm{C}$, 保温 $2 \mathrm{~h}, \mathrm{~N}_{2}$ 流量为 $100 \mathrm{~mL} / \mathrm{min}$. D-PVC-ZnOnm 和S-PVC-ZnOnm采用两 步法制备出的碳材料分别命名为 T-D、T-S; 另外, 采 用上述溶剂分散的方法, 将 $1 \mathrm{~g} \mathrm{PVC}$ 与 $4 \mathrm{~g} \mathrm{ZnOnm}$ 混 合, 两步法制备出碳材料, 命名为T-S4.

\section{2 样品表征}

利用Netzsch STA 449 F5 热重分析仪考察了 O-P、 $\mathrm{O}-\mathrm{D}$ 及 O-S 随着温度变化而发生的质量改变, 实验在 $\mathrm{N}_{2}$ 气氛(流量为 $100 \mathrm{~mL} / \mathrm{min}$ )中进行, 以 $10^{\circ} \mathrm{C} / \mathrm{min}$ 从 $50^{\circ} \mathrm{C}$ 升 温至 $700^{\circ} \mathrm{C}$. 利用有机元素分析仪Vario EL III分析碳材 料产物的C、H元素含量. 采用扫描电子显微镜( SEM, 型号FEI Nova Nano SEM 450)、材料型场发射透射 电子显微镜(TEM, 型号Talos F200X G2)分析产物微观 形貌. 利用拉曼光谱仪 (型号Horiba Jobin Yvon XploRA, 激光的激发波长为 $532 \mathrm{~nm}$ )、X射线光电子 能谱仪(XPS, 型号Kratos AXIS UltraDLD)、傅里叶变 换红外光谱仪(FTIR, 型号Bruker Optics TENSOR 37) 分析产物官能团, 如 $\mathrm{C}=\mathrm{C} 、 \mathrm{C}-\mathrm{Cl} 、 \mathrm{C}=\mathrm{O}$ 等; 采用物理 吸附仪Micromeritics ASAP 2460, 进行 $77 \mathrm{~K} \mathrm{~N}_{2}$ 吸附解吸等温分析, 测量前, 样品在 $150^{\circ} \mathrm{C}$ 脱气 $6 \mathrm{~h}$, 通过 Brunauer-Emmett-Teller (BET)方程计算比表面积, 根 据Barrett-Joyner-Halenda (BJH)模型, 从等温线吸附分 支曲线中采集孔径分布图.

产物在X射线粉末衍射仪(XRD, 型号Bruker D8 advance)上获得XRD光谱, 射线为 $\mathrm{Cu} \mathrm{K} \alpha$. 基于XRD谱 图进行定量计算 ${ }^{[12]}$, 可通过布拉格方程(式1)得到碳材 料的石墨层(002)晶面间距 $d_{002}$, 通过谢乐公式(式2)计 算石墨晶体(100)晶面方向和(002)晶面方向的平均尺 寸 $L_{\mathrm{c}} 、 L_{\mathrm{a}}$ 和石墨层平均堆叠层数 $n$ (式3):

$d_{002}=\frac{\lambda}{2 \sin \theta} \times 10$

$L=\frac{K \lambda}{\frac{\pi}{180^{\circ}} B_{2 \theta} \cos \theta} \times 10$

$n=\frac{L_{\mathrm{c}}}{d_{002}}$ 
式中， $d_{002}$ 是碳材料的石墨层(002)晶面间距， $\AA ; \lambda$ 是 $X R D$ 测试所用 $X$ 射线的波长, $0.154 \mathrm{~nm} ; \theta$ 是衍射角， $B_{2 \theta}$ 是相应衍射峰的半峰宽; $K$ 为无量纲常数, 在计算 $L_{\mathrm{c}}$ 和 $L_{\mathrm{a}}$ 时分别取 0.91 和 $1.84 ; n$ 是石墨层平均堆叠层数, 无 量纲.

\section{3 电化学测试}

为制备工作电极, 将碳材料与纳米导电炭黑、聚 四氟乙烯粉末按 $8: 1: 1$ 的质量比混合, 之后在混合物中 加入 $1 \mathrm{~mL}$ 分析纯乙醇, 超声处理 $30 \mathrm{~min}$ 使其混合均匀. 将混合物负载到泡沫镍片(面积: $1 \mathrm{~cm} \times 1 \mathrm{~cm}$, 质量: $2.0 \mathrm{mg}$ )上，超声混合 $30 \mathrm{~min}$ 后取出并放入 $55^{\circ} \mathrm{C}$ 电热恒 温鼓风干燥箱内烘干. 使用压片机, 以 $20 \mathrm{MPa}$ 的压力 将负载了碳材料的泡沫镍(充当支撑和电流收集器)压 片. 用蒸馏水冲洗负载后的镍片, 洗去表面未附着牢固 的碳粉末, 随后放入 $55^{\circ} \mathrm{C}$ 烘箱中烘干, 烘干后称重并在 $\mathrm{KOH}$ 溶液中浸泡过夜, 用于电化学测试. 以碳材料为 基准, 计算比电容.

在三电极体系的电化学工作站上, 以 $6 \mathrm{~mol} / \mathrm{L}$ 的 $\mathrm{KOH}$ 溶液作为电解液, 负载了碳材料的镍片作为工作 电极, 标准 $\mathrm{Ag} / \mathrm{AgCl}$ 电极作为参比电极, 铂片作为对电 极, 采用循环伏安法(cyclic voltammetry, CV)、恒流充 放电法(galvanostatic charge-discharge, GCD)和电化学 阻抗谱法(electrochemical impedance spectroscopy, EIS), 测试碳材料的电化学性能. 因为 $\mathrm{GCD}$ 的放电曲 线不是直线, 所以采用积分法(式4)计算比电容 ${ }^{[13]}$ :

$$
C=\frac{2 i_{\mathrm{m}} \int V \mathrm{~d} t}{\left.V^{2}\right|_{V_{\mathrm{i}}} ^{V_{\mathrm{f}}}}
$$

式中, $C$ 为比电容, $\mathrm{F} / \mathrm{g} ; i_{\mathrm{m}}$ 为电流密度, $\mathrm{A} / \mathrm{g} ; \int V \mathrm{~d} t$ 为放电 曲线的电流积分面积, $\mathrm{AV} \mathrm{s} ; V_{\mathrm{i}}$ 与 $V_{\mathrm{f}}$ 分别为电压窗口的 起始与终点值, V.

\section{3 结果与讨论}

\subsection{PVC 基碳材料产量分析}

纯PVC、D-PVC-ZnOnm和S-PVC-ZnOnm采用一 步法制备获得碳材料(O-P、O-D、O-S)的热重曲线如 图1所示.

添加ZnOnm可改变PVC的热解行为，大大提高碳 材料的固定碳含量, 且溶剂分散法的作用大于干混法.

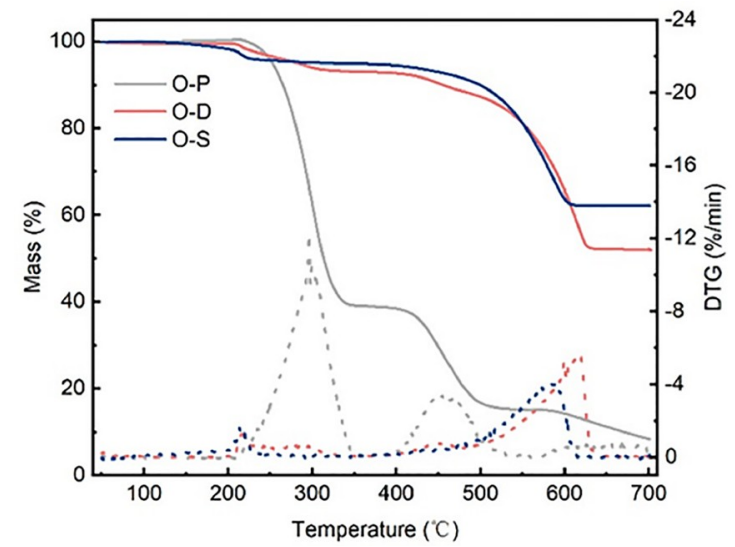

图 1 PVC和PVC-ZnOnm一步法制备获得碳材料的热重曲 线 $\left(100 \mathrm{~mL} / \mathrm{min} \mathrm{N}_{2}\right.$ ) (网络版彩图)

Figure 1 Thermogravimetric curves of the carbon materials obtained from one-stage pyrolysis of PVC and PVC-ZnOnm $\left(100 \mathrm{~mL} / \mathrm{min}_{2}\right)$ (color online).

$\mathrm{PVC}$ 在 $250 \sim 350^{\circ} \mathrm{C}$ 时热分解, 氯原子从分子链上脱离, 生成 $\mathrm{HCl} 、 \mathrm{H}_{2} 、 \mathrm{C} 3$ 以下的气态烃和固态的脱氯多烯烃 (De-Cl PVC $)^{[3]}$. De-Cl PVC含有大量 $\mathrm{sp}^{2}$ 杂化的碳碳双 键. 加热到更高温时, 发生De-Cl PVC的分解和多烯与 碳的交联, 生成稳定的苯环结构的残碳(占PVC质量的 8.39\%). 溶剂分散反应物S-PVC-ZnOnm的分解经历了 两个阶段：在 $193^{\circ} \mathrm{C}<T<235^{\circ} \mathrm{C}$ 时, $\mathrm{PVC}$ 与 $\mathrm{ZnOnm}$ 反应, 生成水和 $\mathrm{ZnCl}_{2}$, 未生成 $\mathrm{HCl}^{[14]}$; 在 $430^{\circ} \mathrm{C}<T<610^{\circ} \mathrm{C}$ 时, $\mathrm{De}-\mathrm{Cl} \mathrm{PVC}$ 和氯氧化锌 $\left[\mathrm{Zn}_{2} \mathrm{OCl}_{2} \cdot 2 \mathrm{H}_{2} \mathrm{O}\right]$ 分解, 高蒸气压 的 $\mathrm{ZnCl}_{2}$ 挥发 ${ }^{[10,15,16]}$. O-D的TG曲线介于 O-S和O-P中 间, 它与 O-S一样削弱了碳材料的失重峰. $\mathrm{ZnOnm}$ 与 PVC接触面积更大时结合更紧密，第一阶段的起始温 度 $T_{\mathrm{i}}$ 更低, $\mathrm{PVC}$ 热解后的固体残渣产量更大.

将管式炉内固体残渣浸出后烘干，对碳材料称重 并进行元素分析，碳材料的产率即为获得的碳材料与 反应物PVC的比, 以质量百分比 \%表示. 碳材料产率以 及其中的 $\mathrm{C} 、 \mathrm{H} 、 \mathrm{O}$ 元素含量如图2所示, $\mathrm{ZnOnm}$ 增加 了碳材料产率, 且两步法的碳材料产量略高于一步法, $\mathrm{T}-\mathrm{D}$ 的产率为 $46.8 \%$. 加热碳化时, $\mathrm{PVC}$ 的表面首先熔 化和分解, 释放的 $\mathrm{HCl}$ 与 $\mathrm{ZnO}$ 反应生成 $\mathrm{ZnCl}_{2}$ 和 $\mathrm{H}_{2} \mathrm{O}^{[17]}$, 随着温度升高，浸渍在混合物中的 $\mathrm{ZnO}$ 保持固态，而 $\mathrm{ZnCl}_{2}$ 会熔融，在液相中扩散并侵入PVC基质。当温度 上升到PVC分解的第二阶段, 一些解离能较低的弱键 会首先开裂形成挥发性物质，它们从液态扩散到气液 界面. 在扩散过程中，挥发分可在氯化锌的催化下生 
成焦炭和不凝气体 ${ }^{[18]}$ ，使热解油的产量降低 ${ }^{[10]}$. 一步 法中生成的氯盐促进PVC的热分解，降低了碳材料产 率, 碳材料的碳元素含量和 $\mathrm{H} / \mathrm{C}$ 比更低.

\subsection{PVC基碳材料表面形貌分析}

图3是PVC基碳材料的SEM图像，D-PVC-ZnOnm 和S-PVC-ZnOnm制备的碳材料的孔隙结构与纯PVC 制备的碳材料有显著差异. $\mathrm{PVC}$ 直接热解时会发生熔 化坍塌，O-P表面光滑且没有明显的孔隙. O-D和T-D 产物接近球状，直径约为 $100 \mu \mathrm{m}$ ，与PVC原料尺寸一 致. 它们表面光滑, 分布有一定量的可见的孔, 且O-D
的孔道结构看上去更加丰富, 证实了 $\mathrm{ZnO}$ 和 $\mathrm{ZnCl}_{2}$ 的催 化作用。 O-S、T-S和T-S4呈现不规则块状，表面粗粘， 有丰富的孔道结构. 不同颗粒之间的PVC热解碳化后 形成的碳材料连接为一体，由于 $\mathrm{ZnOnm}$ 的支撑作用， 形成三维相互连通的分级多孔结构。这些孔的存在可 以为电极材料提供较大的比表面积，较厚的孔壁有利 于电荷的存储.

通过TEM (图4)进一步观察碳材料, O-D中出现了 由重叠的浴轮层晶格构成的丰富石墨区域，沿着内壁 则出现了许多非晶态结构，显示出由纳米ZnOnm为模 板制成的孔单元的轮廓边缘 ${ }^{[4]}$, 呈现出典型的软碳结
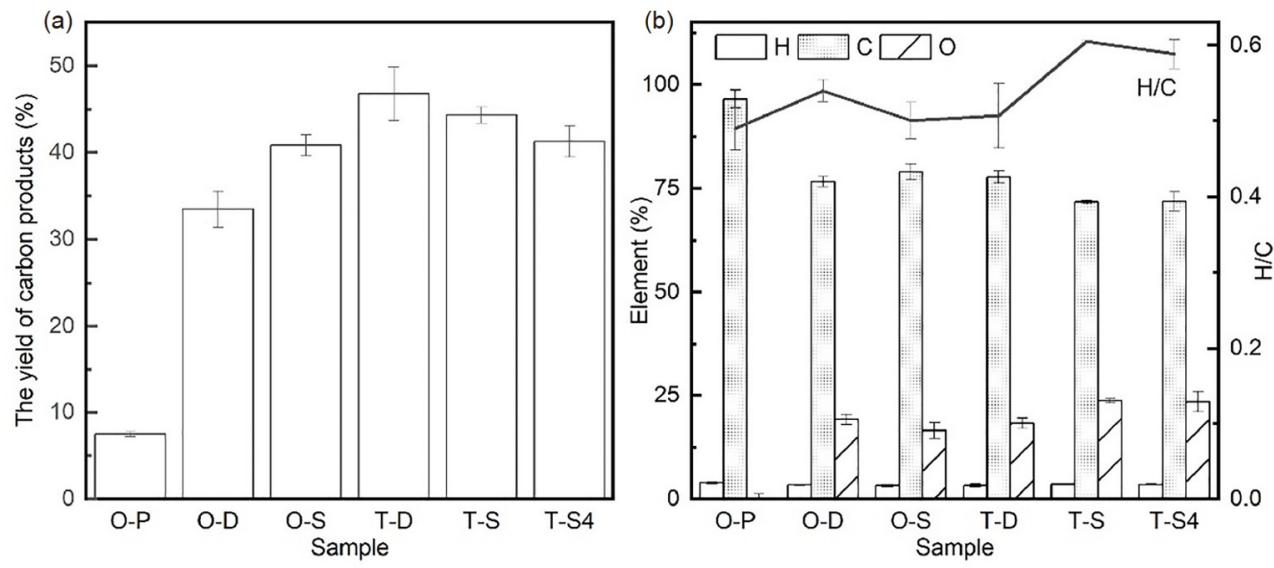

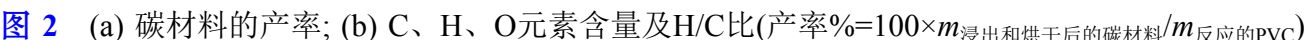

Figure 2 (a) The yield of the carbon materials; (b) $\mathrm{C}, \mathrm{H}, \mathrm{O}$ element contents and $\mathrm{H} / \mathrm{C}$ ratio (The yield of carbon products $\%=100 \times m_{\text {carbon material after }}$ leaching and drying $/ m_{\text {the reactants PVC }}$.
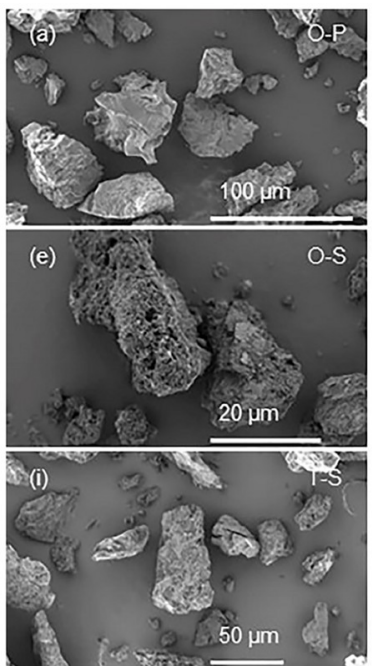
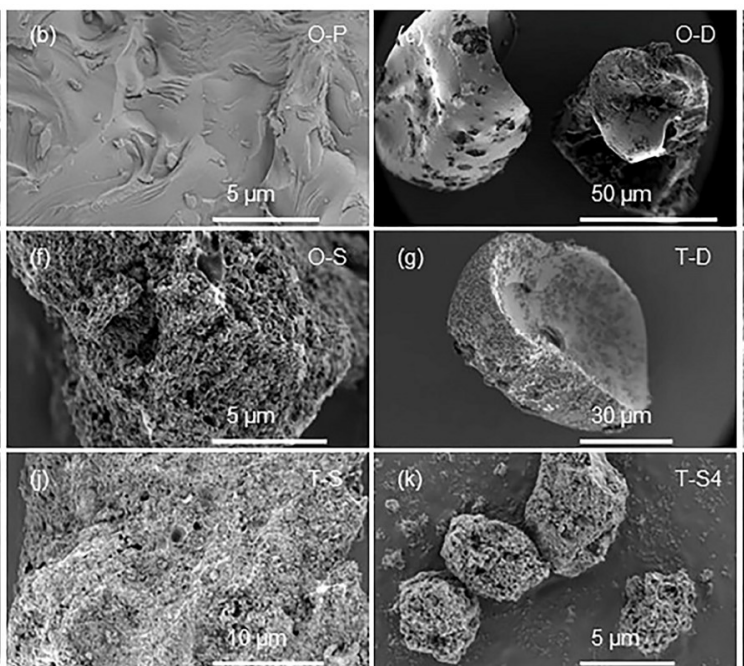

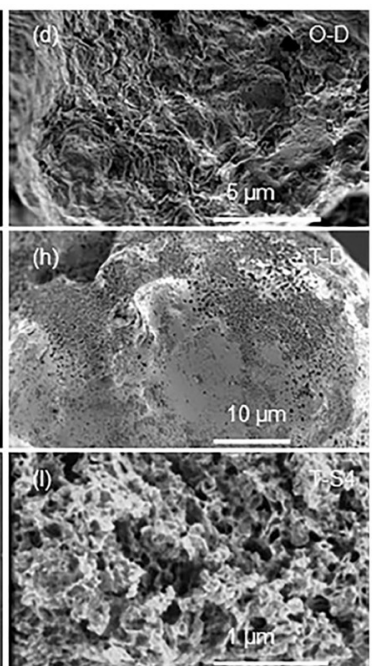

图 3 碳材料SEM图. (a, b) O-P, (c, d) O-D, (e, f) O-S, (g h) T-D, (i, j) T-S, (k, l) T-S4

Figure 3 SEM images of the carbon materials. (a, b) O-P, (c, d) O-D, (e, f) O-S, (g, h) T-D, (i, j) T-S, (k, l) T-S4. 

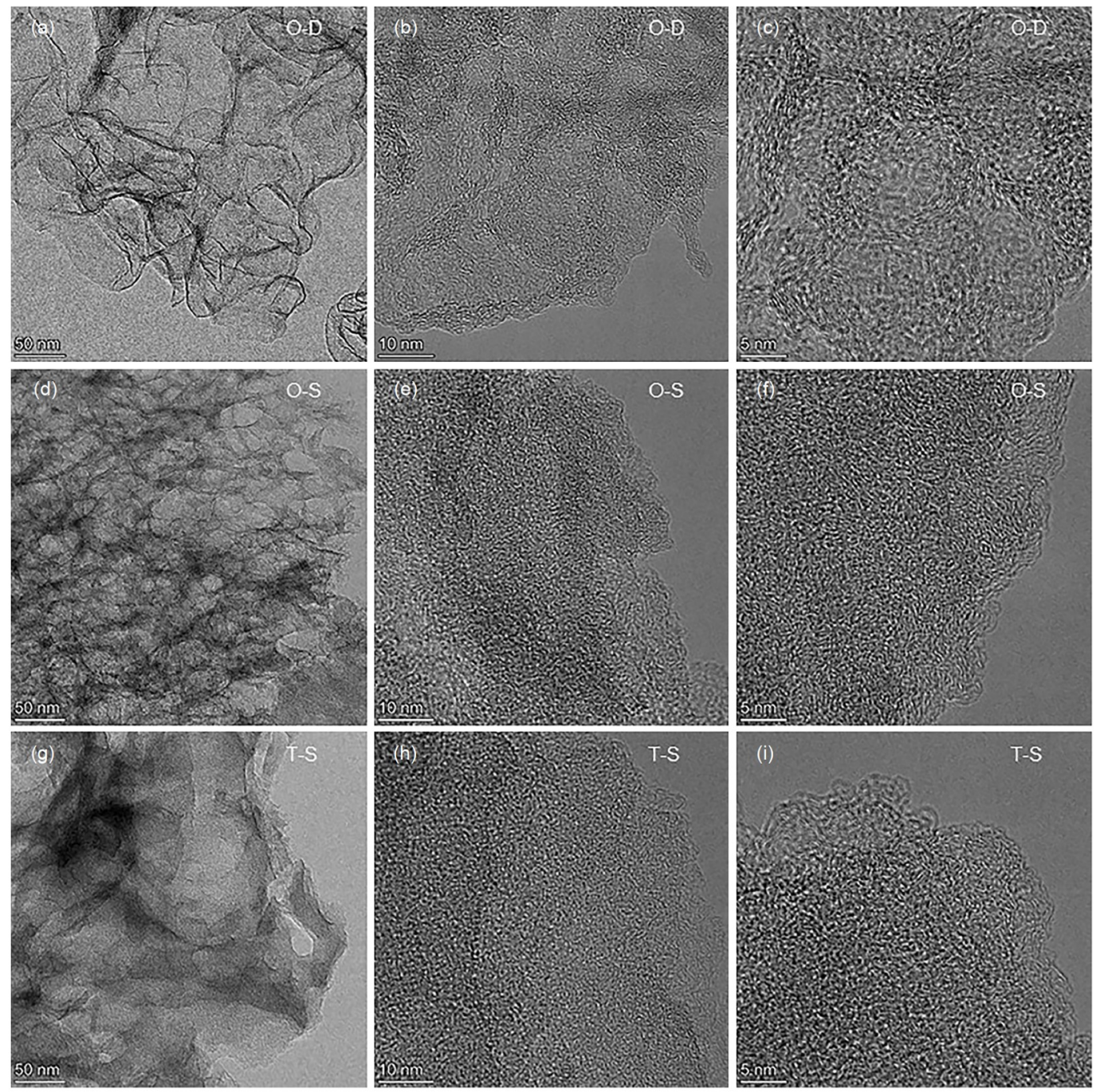

图 4 碳材料TEM图. (a c) O-D, (d f) O-S, (g i) T-S

Figure 4 TEM images of the carbon materials. (a-c) O-D, (d-f) O-S, and (g-i) T-S.

构. 石墨层结构可以快速传递电子, 降低材料的等效串 联电阻 ${ }^{[19,20]}$. 而O-S 和O-S4具有随机分布的石墨层结 构, 表明其无序度较高 ${ }^{[21]}$. 图 $4 \mathrm{c}, \mathrm{f}, \mathrm{i}$ 中可见空白点, 是 样品中相互连通的介孔和微孔，这表明通过催化碳化 成功合成了 HPC.

采用 $\mathrm{N}_{2}$ 吸附-脱附测试确定碳材料的孔道结构, 结 果见图5. 所有碳材料样品的等温线均为I型和IV型, 在 低 $\mathrm{N}_{2}$ 分压段有较多的氮气吸附, 说明碳材料中有一定 量的微孔. 在脱附时, 曲线在0.45 1.0 $P / P_{0}$ 处出现了非 常明显的H4回滞环，是介孔吸附曲线的特征. 从孔径 分布图中可以看出, 除T-D中只有微孔(1 2 nm孔径) 外, O-P和O-D中存在微孔和小介孔(2 5 nm孔径), 所
有溶剂分散S-PVC-ZnOnm制备的碳材料样品中均存 在微孔、小介孔和20 60 $\mathrm{nm}$ 孔径的中介孔和大孔，这 些碳材料呈现出层次性的分级多孔结构. 微孔可以提 高双电层电容, 介孔可以加速电极反应中离子的快速 迁移并改善高电流密度下的功率性能, 同时大孔可用 作电解质的微储库并提供较短的扩散距离, 因此促进 了电解质离子的快速运输 ${ }^{[22,23]}$, 提高电容量. 当能量 密度较高时, 介孔的丰度越大, 电容器的充放电性能 越好; 在低能量密度下, 电容的充放电性能受总孔体 积的影响, 层次化多孔结构的 $\mathrm{HPC}$ 是理想的电容器材 料 ${ }^{[24,25]}$. 表1总结了 $\mathrm{PVC}$ 基碳材料的孔隙结构参数. $\mathrm{T}-\mathrm{D}$ 的比表面积 $\left(S_{\mathrm{BET}}\right)$ 仅为 $74.2 \mathrm{~m}^{2} / \mathrm{g}$, 远小于 $\mathrm{O}-\mathrm{D}$ 的 $S_{\mathrm{BET}}$ 
(161 m²/g). D-PVC-ZnOnm中ZnOnm的模板作用有限, 且一步法制备碳材料时, 随着温度升高到 $550^{\circ} \mathrm{C}$ 以上, $\mathrm{ZnCl}_{2}$ 开始从熔融的反应物中挥发出来, 反应物的进一 步碳化和芳构化导致固体产物尺寸收缩，使微孔和介 孔体积减少 ${ }^{[26,27]}$. 对于 S-PVC-ZnOnm制备的碳材料, $\mathrm{O}-\mathrm{S}$ 的 $S_{\mathrm{BET}}$ 为 $327 \mathrm{~m}^{2} / \mathrm{g}$, 总孔隙体积 $\left(V_{\mathrm{total}}\right)$ 为 $0.50 \mathrm{~cm}^{3} / \mathrm{g}$. 而 T-S 的 $S_{\mathrm{BET}}$ 为 $544 \mathrm{~m}^{2} / \mathrm{g}, V_{\mathrm{total}}$ 为 $0.66 \mathrm{~cm}^{3} / \mathrm{g}$. 在样品制备 阶段，不同颗粒的 PVC被连接为一体，形成镶嵌着 $\mathrm{ZnOnm}$ 的三维结构. $\mathrm{ZnOnm}$ 和PVC接触更加紧密, $\mathrm{ZnOnm}$ 的模板作用更强. 通过酸浸洗涤法去除留在碳 材料中的 $\mathrm{ZnCl}_{2}$ 和 $\mathrm{ZnO}$, 会在碳材料内部结构中产生额 外的微孔和介孔，同时热解过程中 $\mathrm{CO}_{2} 、 \mathrm{H}_{2} \mathrm{O}$ 和 $\mathrm{CH}_{4}$ 等 气体产物的大量释放导致碳上有大量孔隙.

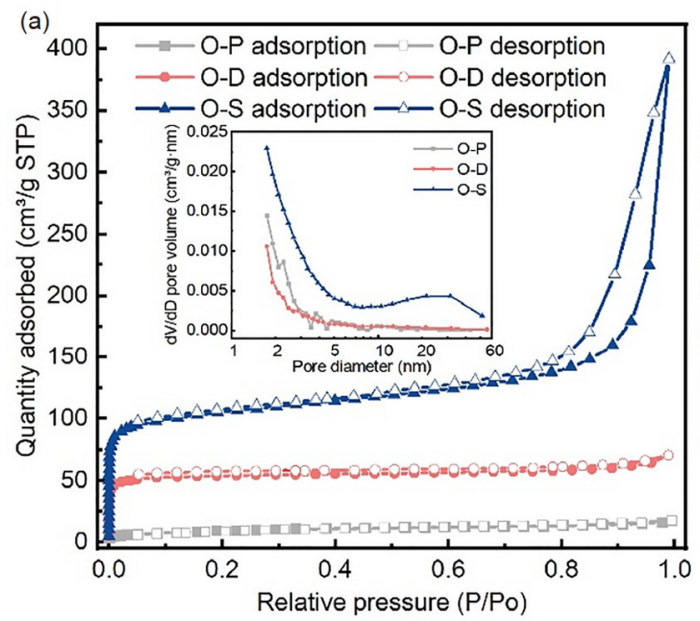

\subsection{PVC基碳材料晶体结构和表面官能团分析}

通过XRD分析PVC基碳材料的晶体结构，由图6a 可知，不同原料和混合方式制备获得碳材料的衍射图 谱差异较大. D-PVC-ZnOnm一步法和两步法制备获得 的碳材料, O-D和T-D的XRD曲线在 $24^{\circ}$ 和 $43^{\circ}$ 左右均有 两个衍射峰, 分别对应石墨的(002)和(100)晶面 ${ }^{[28]}$. 衍 射蜂呈山包形, 说明碳材料均为无定形的石墨结构. SPVC-ZnOnm制备获得的碳材料，在XRD谱图衍射角 $14.5^{\circ}$ 处新增一个衍射峰, 是石墨层被氧化后层间距扩 大造成的 ${ }^{[29]}$. 对比O-S、T-S 和T-S4, 石墨特征衍射峰 $\left(26^{\circ}\right)$ 的强度逐渐减弱, 氧化石墨衍射峰 $\left(14.5^{\circ}\right)$ 增强, 说明石墨化结构被破坏. PVC-ZnOnm热解固体残渣洗 去氯盐后再碳化和增大 $\mathrm{ZnO}$ 的添加量, 均会降低碳材

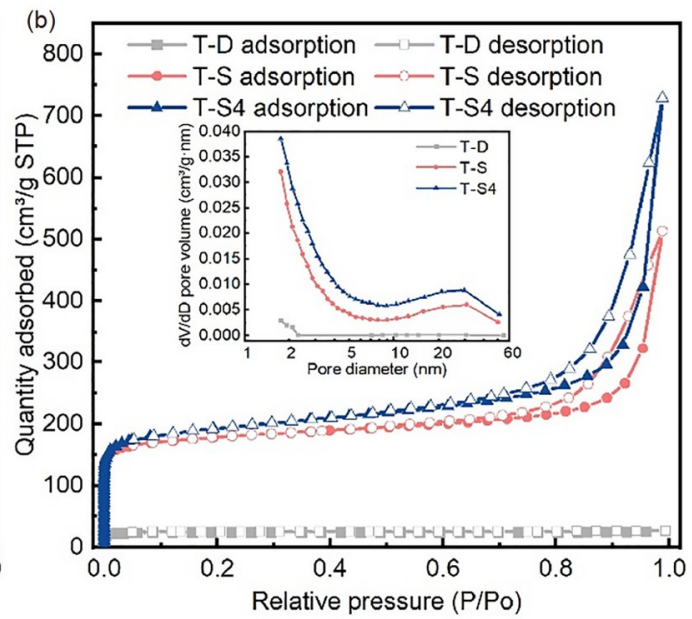

图 5 碳材料的 $\mathrm{N}_{2}$ 吸附等温线, 内插图为孔径分布图. 一步法(a)和两步法(b)制备的碳材料(网络版彩图)

Figure $5 \quad \mathrm{~N}_{2}$ adsorption isotherms of the carbon materials prepared by (a) one-stage method and (b) two-stage method; the insets are pore size distribution figures (color online).

表 1 碳材料 $\mathrm{N}_{2}$ 吸附-脱附测试的计算结果

Table 1 Calculated results from the $\mathrm{N}_{2}$ adsorption-desorption measurements

\begin{tabular}{|c|c|c|c|c|c|c|}
\hline Sample & $S_{\mathrm{BET}}{ }^{\mathrm{a})}\left(\mathrm{m}^{2} / \mathrm{g}\right)$ & $S_{\text {mic }}{ }^{\text {b) }}\left(\mathrm{m}^{2} / \mathrm{g}\right)$ & $V_{\text {total }}{ }^{\mathrm{c})}\left(\mathrm{cm}^{3} / \mathrm{g}\right)$ & $V_{\text {micro }}{ }^{\mathrm{b})}\left(\mathrm{cm}^{3} / \mathrm{g}\right)$ & $D_{\text {aver }}{ }^{\mathrm{d})}(\mathrm{nm})$ & $C(\mathrm{~F} / \mathrm{g})$ \\
\hline O-P & 33.4 & - & 0.025 & - & 3.5 & 36.4 \\
\hline O-D & 161 & 135 & 0.11 & 0.071 & 7.3 & 168 \\
\hline O-S & 327 & 200 & 0.50 & 0.11 & 17.2 & 226 \\
\hline T-D & 74.2 & 68.2 & 0.041 & 0.035 & 5.4 & 140 \\
\hline T-S & 544 & 389 & 0.66 & 0.21 & 16.4 & 150 \\
\hline T-S4 & 599 & 369 & 0.86 & 0.20 & 17.2 & 180 \\
\hline
\end{tabular}

a) 总比表面积 $S_{\mathrm{BET}}$ 用BET法计算; b) 微孔比表面积 $S_{\text {mic }}$ 和体积 $V_{\text {micro }}$ 由t-plot法计算; c) 总孔隙体积 $V_{\text {total }}$ 由 $P / P_{0}=0.95$ 处计算得到; d) 平均孔 径 $D_{\mathrm{aver}}$ 由 $\mathrm{BJH}$ 脱附曲线计算得到 

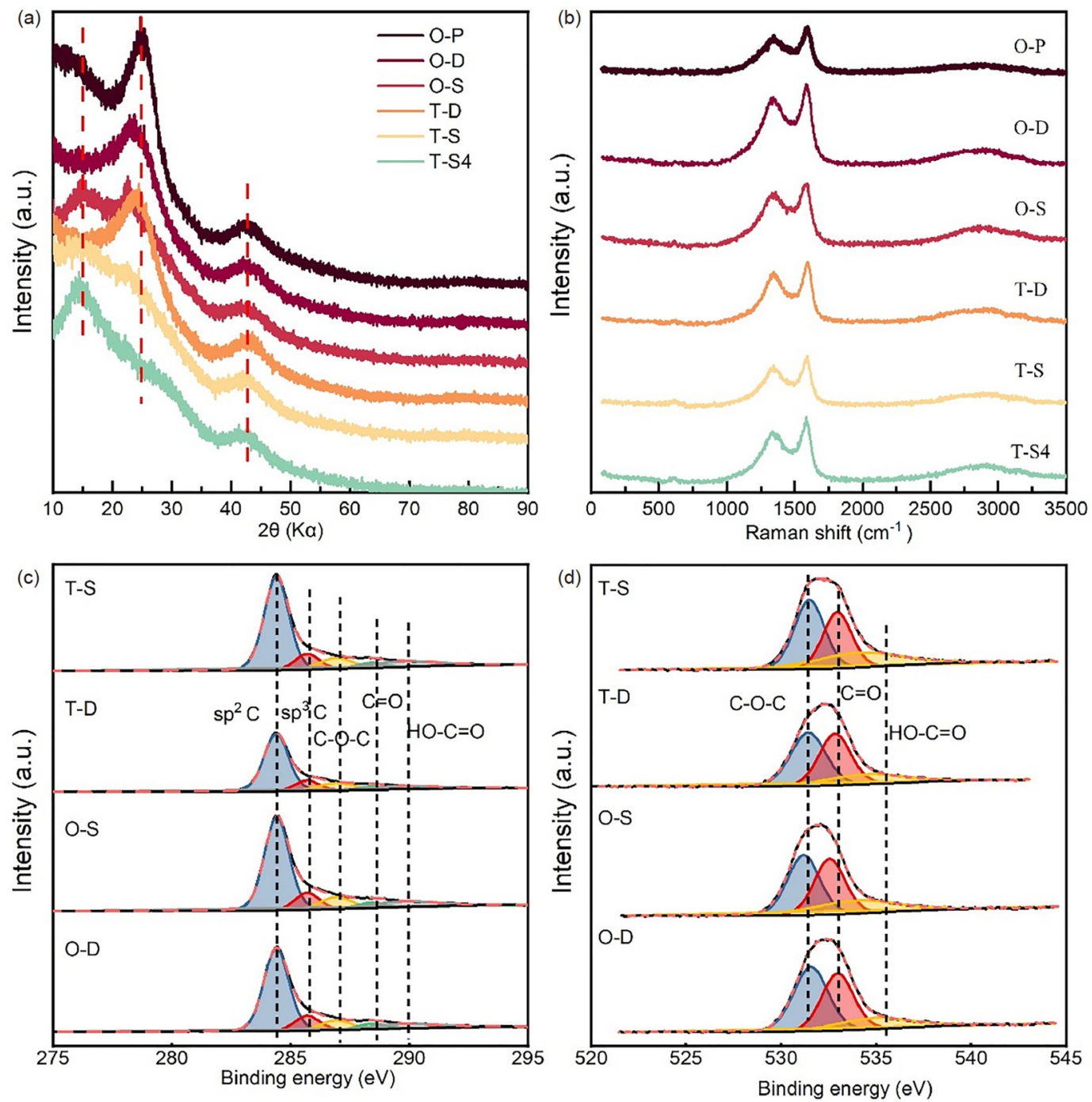

图 6 碳材料的XRD光谱(a), 拉曼光谱(b), XPS的C 1s谱图(c), XPS的O 1s谱图(d) (网络版彩图)

Figure 6 (a) XRD spectra, (b) Raman spectra, (c) XPS spectra of C $1 \mathrm{~s}$ and XPS spectra of O $1 \mathrm{~s}$ (d) of the carbon materials (color online).

料的石墨化程度. 说明 $\mathrm{ZnCl}_{2}$ 促进碳材料的石墨化，而 $\mathrm{ZnO}$ 促进碳材料的氧化. $\mathrm{ZnO}$ 与 PVC的接触面积越大, 氧化作用越强.

基于XRD谱图定量计算了碳材料在石墨(002)晶 面方向的平均尺寸 $L_{\mathrm{a}}$ 、层间距 $d_{002}$ 、(100) 晶面方向平 均尺寸 $L_{\mathrm{c}}$ 和石墨层平均堆叠层数 $n$, 如表 2 所示. $\mathrm{O}-\mathrm{S}$ 的 $d_{002}$ 间距最小 $(3.77 \AA)$, 晶格缺陷减少, 微晶的排列更规 整. 同时 $\mathrm{O}-\mathrm{S}$ 的微晶尺寸 $L_{\mathrm{a}}$ 最大，层面堆叠厚度 $L_{\mathrm{c}}$ 和平 均堆叠层数 $n$ 最小, 其石墨层片尺寸更大, $\pi-\pi *$ 共轭作 用更强, 结构更稳定 ${ }^{[30]}$. 其他碳材料样品的 $d_{002}$ 间距在

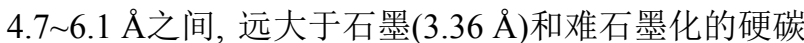

$(4.17 \AA)^{[4]}$, 无序程度更大. S-PVC-ZnOnm制备的碳材 料中, T-S、T-S4的 $d_{002} 、 L_{\mathrm{c}}$ 和 $n$ 比O-S更大, $L_{\mathrm{a}}$ 更小, 说 明两步法去除锌的氯盐不利于石墨化进程. D-PVCZnOnm制备的碳材料中, O-D和T-D的 $L_{\mathrm{a}} 、 d_{002} 、 L_{\mathrm{c}}$ 和 $n$ 差距不大, 因为 $\mathrm{ZnO}$ 等无机物与 $\mathrm{PVC}$ 接触面积更小, 碳 材料的晶体结构基本不变化.

图6b中, 样品均有碳原子晶体的拉曼特征峰: D峰 $\left(1360 \mathrm{~cm}^{-1}\right)$ 代表碳材料中的 $\mathrm{sp}^{3}$ 位点，一般由碳原子晶 格结构缺陷引起; $\mathrm{G}$ 峰 $\left(1585 \mathrm{~cm}^{-1}\right)$ 代表碳材料中的 $\mathrm{sp}^{2}$ 结构 ${ }^{[31]}$. 所有样品的 $G$ 峰均比 $D$ 峰高, 说明它们具有较 好的石墨微晶结构. D峰和 $\mathrm{G}$ 峰的强度比 $\left(I_{\mathrm{D}} / I_{\mathrm{G}}\right)$ 越小, 缺 
表 2 基于XRD、拉曼光谱和XPS的计算结果

Table 2 Calculated results based on XRD, Raman and XPS spectroscopy

\begin{tabular}{cccccccc}
\hline 样品 & $L_{\mathrm{a}}{ }^{\mathrm{a})}(\AA)$ & ${d_{002}{ }^{\mathrm{a})}(\AA)}^{\AA} L_{\mathrm{c}}{ }^{\mathrm{a})}(\AA)$ & $n^{\mathrm{a})}$ & $I_{\mathrm{D}} / I_{\mathrm{G}}{ }^{\mathrm{b})}$ & $\mathrm{C}_{\text {含量 }}{ }^{\mathrm{c})}(\% \mathrm{wt})$ & $\mathrm{O}$ 含量 ${ }^{\mathrm{c})}(\% \mathrm{wt})$ \\
\hline O-P & 4.22 & 5.29 & 9.42 & 1.78 & 0.83 & 90.78 & 9.22 \\
O-D & 6.53 & 4.78 & 7.99 & 1.67 & 0.91 & 90.30 & 9.70 \\
O-S & 13.15 & 3.77 & 3.71 & 0.98 & 0.96 & 89.77 & 10.23 \\
T-D & 6.79 & 4.70 & 8.24 & 1.75 & 0.97 & 89.93 & 10.07 \\
T-S & 2.44 & 6.08 & 12.32 & 2.03 & 0.97 & 90.57 & 9.43 \\
\hline
\end{tabular}

a) XRD的计算结果; b) 基于拉曼光谱的计算结果; c) XPS测试结果

陷更少, 石墨化程度更高. O-P的 $I_{\mathrm{D}} / I_{\mathrm{G}}$ 值最低, 因为其他 PVC-ZnO混合物中的ZnO降低了碳材料的石墨化. SPVC-ZnOnm中的ZnO与PVC接触更紧密，制备获得的 $\mathrm{O}-\mathrm{S}$ 的 $I_{\mathrm{D}} / I_{\mathrm{G}}$ 大于 $\mathrm{O}-\mathrm{D}$, 石墨化程度更低. 两步法制备的 碳材料的 $I_{\mathrm{D}} / I_{\mathrm{G}}$ 大于一步法, 碳材料的无序度更大, 此结 果与XRD的分析一致.

通过XPS分析碳材料的表面化学性能，样品中均 检测到 $\mathrm{C} 、 \mathrm{O}$ 两种元素，而 $\mathrm{Cl}$ 元素低于检测限值，说明 $\mathrm{ZnOnm}$ 基本将PVC中的有机氯脱除. 通过CasaXPS软 件对XPS谱图解谱分析，得到 C 1s、O 1s 的高分辨率 XPS谱图(图6c, d). C 1s 被分为五个单峰, 分别对应 $\mathrm{sp}^{2}$ $\mathrm{C}(284.4 \mathrm{eV}) 、 \mathrm{sp}^{3} \mathrm{C}(285.7 \mathrm{eV}) 、 \mathrm{C}-\mathrm{O}-\mathrm{C}(286.9 \mathrm{eV}) 、$ $\mathrm{C}=\mathrm{O}(288.4 \mathrm{eV})$ 和 $\mathrm{C}-\mathrm{OH}(289.8 \mathrm{eV})^{[30]}$. O 1s 拟合峰的 位置分别为 $531.8 、 532.9$ 和 $534.3 \mathrm{eV}$, 分别对应 $\mathrm{C}=\mathrm{O}$ 、 $\mathrm{HO}-\mathrm{C}=\mathrm{O}$ 和 $\mathrm{C}-\mathrm{O}-\mathrm{C}^{[32]}$. $\mathrm{ZnO}$ 使碳材料生成了大量的含 氧官能团(oxygen functional groups, OFGs). 在碳材料 表面引入OFGs可以产生噟电容, 同时改善其在电解液 中的润湿性和吸附性，加速电解液电荷在碳材料表面 的传递速度 ${ }^{[33,34]}$. 与 T-D相比, $\mathrm{O}-\mathrm{D}$ 的 $\mathrm{sp}^{2} \mathrm{C}$ 峰增强, $\mathrm{sp}^{3}$ $\mathrm{C} 、 \mathrm{C}-\mathrm{O}$ 和 $\mathrm{C}=\mathrm{O}$ 峰减弱，说明一步法中 $\mathrm{ZnCl}_{2}$ 的存在可 以提高碳材料的石墨化程度，同时减少表面官能团含 量, 这与XRD和拉曼光谱的结论一致.

通过FTIR分析碳材料的表面官能团(图7). 在 $3000 \sim 3700 \mathrm{~cm}^{-1}$ 范围内观察到较强和较宽的吸收带, 与羟基 $(-\mathrm{OH})$ 的拉伸振动有关. 在 $2917 、 2852$ 和 $1631 \mathrm{~cm}^{-1}$ 的吸收峰分别属于 $\mathrm{sp}^{2}$ 的 $\mathrm{C}-\mathrm{H}$ 伸缩振动、 $\mathrm{sp}^{3}$ 的 $\mathrm{C}-\mathrm{H}$ 伸缩振动和烯烃 $\mathrm{C}=\mathrm{C}$ 的伸缩振动. $930 \sim 1220 \mathrm{~cm}^{-1}$ 范围内, 对应不对称 $\left(1160 \mathrm{~cm}^{-1}\right)$ 和对称 $\left(1110 \mathrm{~cm}^{-1}\right)$ 的 $\mathrm{C}-\mathrm{O}-\mathrm{C}$ 伸缩振动和 $\mathrm{C}-\mathrm{OH}\left(1050 \mathrm{~cm}^{-1}\right)$ 伸缩振动 ${ }^{[32]}$. 这 些特征峰的存在证明了制备碳材料的过程中引入了羟
基、环氧基等OFGs，丰富的OFGs在电容性能中起着 重要作用 ${ }^{[35]}$. 碳材料表面的OFGs可以和质子发生可 逆的电化学反应, 由此产生赝电容. 增加OFGs还可以 增加碳材料的表面润湿性 ${ }^{[36,37]}$, 进一步增加离子在表 面的迁移速率，使离子更容易形成双层，进行插入/脱 插入反应 ${ }^{[38]}$. 然而, 体积庞大的OFGs会破坏 $\mathrm{sp}^{2}$ 碳网络, 降低电导率, 过量的OFGs也会影响离子在电解质中的 扩散 ${ }^{[39]}$. 因此, 合理调节碳材料表面的OFGs是至关重 要的.

\subsection{PVC基碳材料电化学性能测试}

对制备的碳材料进行电化学表征. 图 $8 \mathrm{a}, \mathrm{b}$ 为碳材 料在 $50 \mathrm{mV} / \mathrm{s}$ 下的 $\mathrm{CV}$ 曲线和在 $1 \mathrm{~A} / \mathrm{g}$ 下的 $\mathrm{GCD}$ 曲线. $\mathrm{O}-\mathrm{S}$ 的 CV曲线存在一峰宽较大的氧化还原峰, 可归结

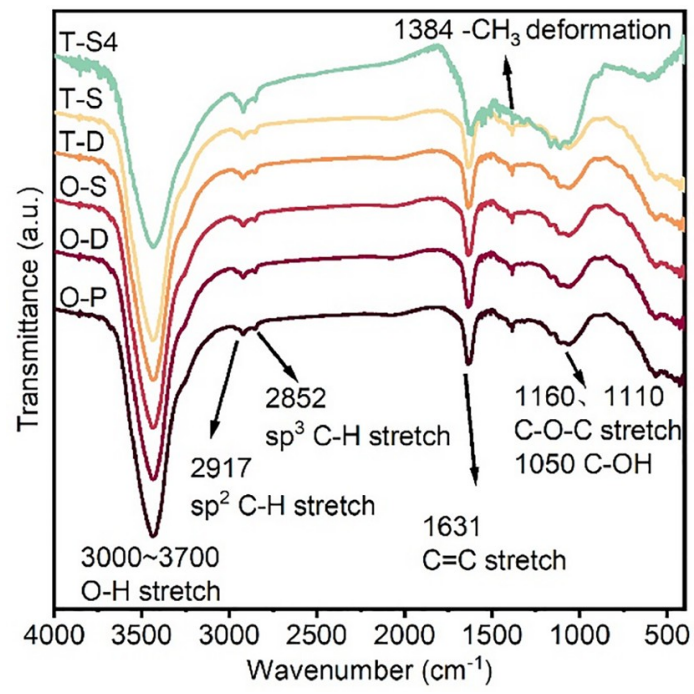

图 7 碳材料的FTIR谱图(网络版彩图)

Figure 7 FTIR spectra of the carbon materials (color online). 

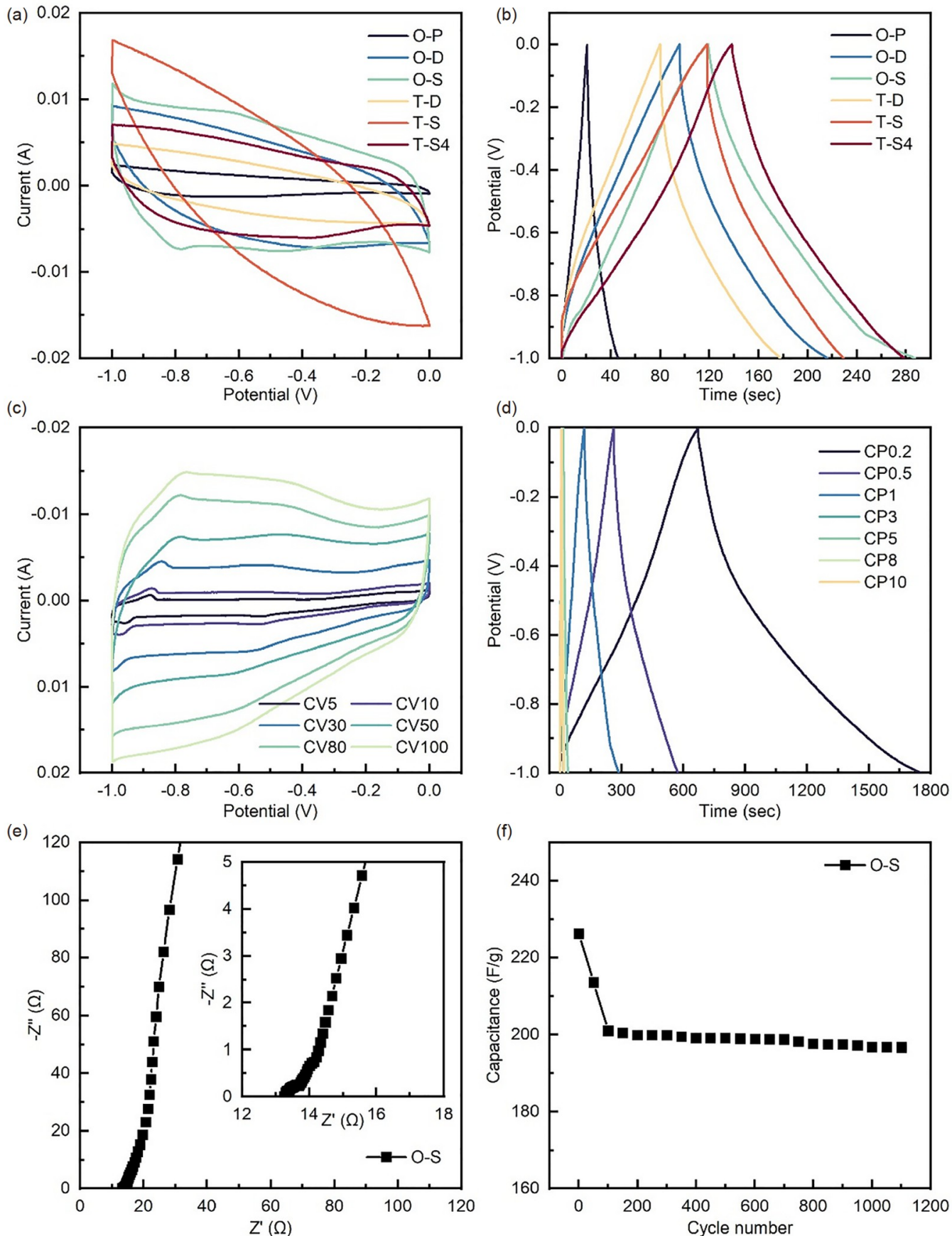

图 8 碳材料 $50 \mathrm{mV} / \mathrm{s}$ 的 CV曲线(a), $1 \mathrm{~A} / \mathrm{g}$ 的 $\mathrm{GCD}$ 曲线(b); (c) 不同扫描速率下 $\mathrm{O}-\mathrm{S}$ 的CV曲线; (d) 不同电流密度下 $\mathrm{O}-\mathrm{S}$ 的 $\mathrm{GCD}$ 曲 线及其倍率曲线; (e) O-S的电化学阻抗谱图; (f) O-S在 $10 \mathrm{~A} / \mathrm{g}$ 条件下 1100 次循环的循环稳定性. 所有测试在三电极系统中进行 (网络版彩图)

Figure 8 (a) CV curves of the carbon materials at $50 \mathrm{mV} / \mathrm{s}$; (b) GCD curves of the carbon materials at $1 \mathrm{~A} / \mathrm{g}$; (c) $\mathrm{CV}$ of O-S at different scan rates; (d) GCD and rate performance of O-S at different current densities; (e) electrochemical impedance spectrogram of O-S; (f) cycling stability of O-S at $10 \mathrm{~A} / \mathrm{g}$ for 1100 cycles. All electrochemical experiments were performed in a three-electrode system (color online).

为材料中 OFGs所引起的法拉第噟电容效应 ${ }^{[40]}$. CV曲 线中其他样品没有明显的氧化还原峰，主要表现为双
电层电容器. 由于极化内阻的存在，双电层电容器在 电化学窗口的CV曲线会偏离标准的矩形. T-D和T-S的 
$\mathrm{CV}$ 曲线变形严重, 倍率性不佳. 孔内离子传输电阻和 扩散距离会对材料的倍率特性产生重要影响. 根据前 文测试结果, 它们的离子传输距离 $L_{\mathrm{c}}$ 较大, 石墨化程度 低即电子传递速度慢, 等效串联电阻较大, 不能完全满 足超级电容器材料的需求 ${ }^{[41]}$. 双电层电容器的 $\mathrm{GCD}$ 曲 线是对称三角形, 样品中有一定的噟电容, 使 $\mathrm{GCD}$ 曲 线产生形状的偏移, 呈准对称三角形 ${ }^{[42]}$. 根据 $\mathrm{GCD}$ 曲线 计算得到比电容 $C$ (表1), 一步法制备的O-P、O-D、O-S 的比电容分别为 $36.4 、 168$ 和 $226 \mathrm{~F} / \mathrm{g}$. 两步法制备的的 T-D、T-S、T-S4的比电容分别为 $140 、 150$ 和 $180 \mathrm{~F} / \mathrm{g}$. 相比 $\mathrm{O}-\mathrm{P}, \mathrm{ZnO}$ 的加入大幅度提高了碳材料的比电 容, 这是因为碳材料的 $S_{\mathrm{BET}}$ 和表面官能团增加. 两步法 制备的T-S4比表面积和孔容最大，但是对大多数多孔 材料, 比电容的增加并不只和 $S_{\mathrm{BET}}$ 及孔容有关 ${ }^{[43]}$, 且比 电容与 $S_{\mathrm{BET}}$ 并没有线性关系 ${ }^{[44]}$. 为实现高容量和良好 的倍率特性, 电容器材料需要有大孔作为离子缓冲池, 中孔提供更短的离子传输距离, 微孔吸附电荷以及石 墨的碳结构来提升材料导电性 ${ }^{[41]}$. 同时, 噟电容表面 能发生快速、可逆的氧化还原反应，可以大幅度提高 双电层电容器的容量. O-S 的比电容远大于两步法制 备的碳材料和 O-D, 是其多孔结构、石墨化程度与 OFGs综合作用的结果.

图8c, d分别是O-S在不同扫描速率下的 CV曲线、 不同电流密度下的 $\mathrm{GCD}$ 曲线及其倍率性能. $\mathrm{O}-\mathrm{S}$ 的 $\mathrm{CV}$ 曲线有准矩形特点和氧化还原峰, $\mathrm{GCD}$ 曲线为准对称 三角形，说明其不仅具有双电层电容的特性，还有赝 电容的特征. 在扫描速度为 $100 \mathrm{mV} / \mathrm{s}$ 时 CV曲线仍然接 近矩形, 表明O-S比较利于电解质离子的快速扩散, 具 有杰出的电容性能. 在 $0.2 、 0.5 、 1 、 3 、 5 、 8$ 和 $10 \mathrm{~A} / \mathrm{g}$ 电流密度下, O-S 的比电容分别为 $298 、 207 、 226$ 、 21、138、121和 $115 \mathrm{~F} / \mathrm{g}$. 在 $10 \mathrm{~A} / \mathrm{g}$ 的电流密度下保有 在 $1 \mathrm{~A} / \mathrm{g}$ 电流密度下比电容的 $51.04 \%$. 这种电容量的降 低, 一方面因为电流密度的增加导致较短的充放电时
间不足以实现较小孔径内部的离子扩散和吸附 ${ }^{[45]}$, 另 一方面是因噟电容作用减弱. 三电极系统 $1 \mathrm{~A} / \mathrm{g}$ 的电流 密度下测试了 $\mathrm{O}-\mathrm{S}$ 的长期使用性能, 结果如图8 8 所示. 经过 1100 次的充放电循环, O-S 保持 $87 \%$ 的比电容量. 1200 次后, 电容失活. 通过EIS测定材料的阻抗性质, 分析其等效串联电阻ESR. ESR由内电阻(电解液的离 子电阻和电极材料本征阻抗)和电极-电解液界面电阻 组成. O-S 的Nyquist曲线如图8e所示, 在低频区域呈直 线, 表现为典型的双电层电容. 电解液和电极材料的欧 姆电阻较高, 为 $13.36 \Omega$, 不利于电子在电极内部转移, 也不利于提高比电容量和比电容在充放电中的稳定性 和倍率性能. 这一方面是因为 $\mathrm{O}-\mathrm{S}$ 的石墨化程度有限, 导电性不高; 另一方面, O-S的微孔含量较高, 不利于 电解液的有效扩散. 因此, 采用S-PVC-ZnOnm和一步 法制备PVC基碳材料的过程中，控制催化剂的种类与 尺寸, 是改善碳材料孔结构和石墨化性质的重要因素, 有利于提高碳材料的电容特性.

\section{4 结论}

为实现 PVC中碳元素的高值化利用, 本研究采用 $\mathrm{ZnO}$ 作为催化剂和碳化模板, 热解PVC制备HPC, 并将 其用于电容器材料, O-S的比电容 $(1 \mathrm{~A} / \mathrm{g}$ ) 达到 $226 \mathrm{~F} / \mathrm{g}$. 在没有活化剂的情况下, HPC产率高达 $46.8 \%$. 研究发 现, $\mathrm{ZnOnm}$ 会降低碳材料的石墨化程度，同时在碳材 料表面引入 $\mathrm{OFGs}$, 而 $\mathrm{ZnCl}_{2}$ 的作用与之相反. 然而, 碳 材料O-S的微孔含量较高, 且导电性被OFGs影响, 使得 其用作电容的内电阻较高为 $13.36 \Omega$, 限制了电容量的 增长与倍率性能. 采用溶剂分散法和一步法制备PVC 基碳材料的过程中，后续可控制催化剂的种类与尺寸 改善碳材料的孔结构和石墨化性质，采用氨流法等提 高碳材料的电容特性. 该研究为PVC基 $\mathrm{HPC}$ 的设计提 供了参考.

\section{参考文献}

1 Europe P. Plastics-the facts 2020: an analysis of European plastics production, demand and waste data. Technical Report, Association of Plastics Manufacturers, 2020

2 Geyer R, Jambeck JR, Law KL. Sci Adv, 2017, 3: e1700782

3 Miranda R, Pakdel H, Roy C, Vasile C. Polym Degrad Stab, 2001, 73: 47-67

4 Liu K, Qian M, Fan L, Zhang S, Zeng Y, Huang F. Carbon, 2020, 159: 221-228

5 Chen XY, Cheng LX, Deng X, Zhang L, Zhang ZJ. Ind Eng Chem Res, 2014, 53: 6990-6997 
6 Cheng LX, Zhang L, Chen XY, Zhang ZJ. Electrochim Acta, 2015, 176: 197-206

7 Liu F, Wang L, Bradley R, Zhao B, Wu W. RSC Adv, 2019, 9: 29414-29423

8 Chang Y, Pang Y, Dang Q, Kumar A, Zhang G, Chang Z, Sun X. ACS Appl Energy Mater, 2018, 1: 5685-5693

9 Tang C, Wang YZ, Zhou Q, Zheng L. Polym Degrad Stab, 2003, 81: 89-94

10 Masuda Y, Uda T, Terakado O, Hirasawa M. J Anal Appl Pyroly, 2006, 77: 159-168

11 Uddin MA, Sakata Y, Shiraga Y, Muto A, Murata K. Ind Eng Chem Res, 1999, 38: 1406-1410

12 Sharma A, Kyotani T, Tomita A. Carbon, 2000, 38: 1977-1984

13 Mai LQ, Minhas-Khan A, Tian X, Hercule KM, Zhao YL, Lin X, Xu X. Nat Commun, 2013, 4: 2923

14 Kosuda T, Okada T, Nozaka S, Matsuzawa Y, Shimizu T, Hamanaka S, Mishima S. Polym Degrad Stab, 2012, 97: 584-591

Zhang B, Yan XY, Shibata K, Uda T, Tada M, Hirasawa M. Mater Trans JIM, 2000, 41: 1342-1350

Lee GS, Song YJ. Miner Eng, 2007, 20: 739-746

Al-Harahsheh M, Al-Nuairat J, Al-Otoom A, Al-Hammouri I, Al-Jabali H, Al-Zoubi M, Abu Alasal S. J Environ Chem Eng, 2019 , 7: 102856

Hu J, Shen D, Wu S, Xiao R. J Anal Appl Pyroly, 2017, 127: 444-450

Chang B, Guo Y, Li Y, Yin H, Zhang S, Yang B, Dong X. J Mater Chem A, 2015, 3: 9565-9577

Deng J, Xiong T, Xu F, Li M, Han C, Gong Y, Wang H, Wang Y. Green Chem, 2015, 17: 4053-4060

Harris PJF. Int Mater Rev, 1997, 42: 206-218

Fuertes AB, Sevilla M. ACS Appl Mater Interfaces, 2015, 7: 4344-4353

Li YT, Pi YT, Lu LM, Xu SH, Ren TZ. J Power Sources, 2015, 299: 519-528

Gou G, Huang F, Jiang M, Li J, Zhou Z. Renew Energy, 2020, 149: 208-216

Sudhan N, Subramani K, Karnan M, Ilayaraja N, Sathish M. Energy Fuels, 2017, 31: 977-985

Ahmadpour A, Do DD. Carbon, 1996, 34: 471-479

Yue Z, Mangun CL, Economy J. Carbon, 2002, 40: 1181-1191

Lu L, Sahajwalla V, Kong C, Harris D. Carbon, 2001, 39: 1821-1833

Jeong HK, Lee YP, Lahaye RJWE, Park MH, An KH, Kim IJ, Yang CW, Park CY, Ruoff RS, Lee YH. J Am Chem Soc, 2008, 130: 1362-1366

Chiappe G, Louis E, San-Fabián E, Vergés JA. J Phys-Condens Matter, 2015, 27: 463001

Eda G, Chhowalla M. Adv Mater, 2010, 22: 2392-2415

Yu BY, Kwak SY. J Mater Chem, 2012, 22: 8345-8353

Jiang L, Wang J, Mao X, Xu X, Zhang B, Yang J, Wang Y, Zhu J, Hou S. Carbon, 2017, 111: 207-214

Kerisit S, Schwenzer B, Vijayakumar M. J Phys Chem Lett, 2014, 5: 2330-2334

Wang X, Qin Z, Yang X, Chen Q, Lin Z. Diamond Relat Mater, 2020, 108: 107995

Li X, Jiang Y, Wang P, Mo Y, Lai W, Li Z, Yu R, Du Y, Zhang X, Chen Y. New Carbon Mater, 2020, 35: 232-243

Hwang SY, Lee CH, Lee HR, Son SY, Lee S, Joh HI. Chem Eng Sci, 2021, 231: 116301

Cao H, Peng X, Zhao M, Liu P, Xu B, Guo J. RSC Adv, 2018, 8: 2858-2865

Guo J, Zheng P. Mater Lett, 2018, 214: 134-137

Du Q, Zheng M, Zhang L, Wang Y, Chen J, Xue L, Dai W, Ji G, Cao J. Electrochim Acta, 2010, 55: 3897-3903

Wang DW, Li F, Liu M, Lu GQ, Cheng HM. Angew Chem Int Ed, 2008, 47: 373-376

Hu Y, Tong X, Zhuo H, Zhong L, Peng X. ACS Sustain Chem Eng, 2017, 5: 8663-8674

Simon P, Gogotsi Y. Nat Mater, 2008, 7: 845-854

Kim H, Fortunato ME, Xu H, Bang JH, Suslick KS. J Phys Chem C, 2011, 115: 20481-20486

Wei L, Sevilla M, Fuertes AB, Mokaya R, Yushin G. Adv Energy Mater, 2011, 1: 356-361 


\title{
Convert PVC to hierarchical porous carbon material by using $\mathrm{ZnO}$ as a template and catalyst
}

\author{
Tian-Tian Meng ${ }^{1}$, Hua Zhang ${ }^{1,2^{*}}$, Fan Lü ${ }^{1,2}$, Li-Ming Shao ${ }^{1,2}$, Pin-Jing He ${ }^{1,2}$ \\ ${ }^{1}$ College of Environmental Science and Engineering, Tongji University, Shanghai 200092, China \\ ${ }^{2}$ Shanghai Institute of Pollution Control and Ecological Security, Shanghai 200092, China \\ *Corresponding author (email: zhanghua_tj@tongji.edu.cn)
}

\begin{abstract}
During thermal treatment of polyvinyl chloride (PVC) at $250-310^{\circ} \mathrm{C}$, dechlorination reaction occurs and carbon atoms change from $\mathrm{sp}^{3}$ hybridization to $\mathrm{sp}^{2}$ hybridization. As the temperature rises, these carbon atoms of $\mathrm{sp}^{2}$ hybridization are further assembled to form aromatic carbon. The product, carbon material, often has a certain degree of graphitization and high conductivity, thus being an excellent precursor for capacitor electrodes. In the absence of activators, PVC was converted to hierarchical porous carbon material (HPC) with a high yield and a specific capacitance of $226 \mathrm{~F} / \mathrm{g}(1 \mathrm{~A} / \mathrm{g})$ successfully by using nano- $\mathrm{ZnO}$ as a template and catalyst. By comparing the mixing method of $\mathrm{ZnO}$ and $\mathrm{PVC}$, and whether to remove $\mathrm{ZnCl}_{2}$ before high-temperature carbonization, it is found that $\mathrm{ZnO}$ reduced the graphitization degree of the carbon materials, meanwhile introducing oxygen functional groups on the surface of the material, while $\mathrm{ZnCl}_{2}$ had an opposite effect. The pore structure and graphitization properties of HPC can be further improved to enhance the capacitance performance by controlling the type and size of catalysts. This study provides a reference for the design of PVC-based HPC.
\end{abstract}

Keywords: polyvinyl chloride, nano zinc oxide, template carbonization, catalysis, capacitance

doi: $10.1360 /$ SSC-2021-0148 